\title{
FACTORIZATION AND DISCONJUGACY OF THIRD ORDER DIFFERENTIAL EQUATIONS
}

\author{
ANTON ZETTL
}

\begin{abstract}
Sufficient conditions for the factorization of $y^{\prime \prime \prime}+$ $p y^{\prime \prime}+q y^{\prime}+r y$ into a product of first order operators as well as into a product of a first order and a second order operator are given. Factorization into a product of first order factors is known to be equivalent to disconjugacy. These conditions are simple inequalities involving the coefficients.
\end{abstract}

We consider the third order linear differential operator

$$
L y=y^{\prime \prime \prime}+p y^{\prime \prime}+q y^{\prime}+r y
$$

and the associated equation

$$
L y=0,
$$

where $p, q$, and $r$ are real valued continuous functions defined on some interval $I$.

Equation (2) is said to be disconjugate on $I$ if no nontrivial solution has more than two zeros, counting multiplicities, on $I$. A well-known-see [15]-necessary and sufficient condition for disconjugacy of (2) and also for the factorization of (1) of the type $L y=p_{3}\left(p_{2}\left(p_{1}\left(p_{0} y\right)^{\prime}\right)^{\prime}\right)^{\prime}$ with $p_{i}(t) \neq 0$ for $i=0, \cdots, 3$ and $t$ in $I$ is condition

$\left(\mathrm{W}_{12}\right)$ : There exists two linearly independent solutions $y_{1}, y_{2}$ of (2) such that $y_{2}>0$ and $W\left(y_{1}, y_{2}\right)>0$ where $W\left(y_{1}, y_{2}\right)=y_{1} y_{2}^{\prime}-y_{2} y_{1}^{\prime}$.

It is also known - see [19] — that a necessary and sufficient condition for the factorization of (1) into a product $P Q$ where $P$ is a first order operator of the type $P y=r_{1} y^{\prime}+r_{0} y$ with $r_{1}(t) \neq 0$ and $Q$ is a second order operator of the type $Q y=q_{2} y^{\prime \prime}+q_{1} y^{\prime}+q_{0} y$ with $q_{2}(t) \neq 0$ is condition

$\left(\mathrm{W}_{2}\right)$ : There exist two linearly independent solutions $y_{1}, y_{2}$ of (2) such that $W\left(y_{1}, y_{2}\right)>0$.

Moreover, given condition $\left(\mathrm{W}_{2}\right), Q$ can be chosen such that $y_{1}$ and $y_{2}$ are linearly independent solutions of $Q y=0$.

Received by the editors January 14, 1971 and, in revised form, April 20, 1971.

AMS 1970 subject classifications. Primary 34A30, 34A05; Secondary 34A01.

Key words and phrases. Ordinary differential equations, disconjugacy criteria, factorization of differential operators, Frobenius factorization, Pólya's property "W," Wronskians, oscillation or nonoscillation of solutions, zeros of solutions, third order linear homogeneous differential equations, Tchebycheff property of solutions. 
In this paper we obtain conditions on the coefficients sufficient for $\left(\mathrm{W}_{12}\right)$ to hold and also for $\left(\mathrm{W}_{2}\right)$. Our conditions for $\left(\mathrm{W}_{12}\right)$ include those recently obtained by $\operatorname{Kim}[10]$. The conditions for $\left(W_{2}\right)$ imply a result of Kim [10, Theorem 1] under a weaker hypothesis. In addition our work establishes a natural framework for the interpretation of some of these results.

For other recent work on disconjugacy or oscillatory behavior of solutions of third order equations see Hanan [6], Azbelev and Caljuk [1], Lazer [11], Dolan [4], Barrett [2], and Jackson [9]. A summary of results on oscillation theory and disconjugacy conditions of third order equations as well as an extensive bibliography can be found in a recent paper of Barrett [3].

For some recent work on $n$th order linear differential equations see Hartman [7] and [8], Willett [16], [17], and [18], Levin [12], Nehari [14], and Fink [5].

We will use the notation $X \geqq 0$ for a matrix or vector $X$ to mean that each component is nonnegative. Similarly $X>0$ will mean that each component of $X$ is strictly positive.

Several of our results make use of

LEMMA 1. Let $F$ be an $n \times n$ matrix of continuous real valued functions. If, for $t \geqq \alpha, F(t) \geqq 0$ and $Y^{\prime}(t)=F(t) Y(t)$ with $Y(\alpha)>0$, then $Y(t) \geqq 0$ for $t>\alpha$.

Proof. Suppose some component of $Y$ is negative to the right of $\alpha$. Then there is some $t_{0}>\alpha$ such that, for some $1 \leqq i \leqq n, y_{i}\left(t_{0}\right)=0$ and every component of $y$ is strictly positive in $\left[\alpha, t_{0}\right)$. There is a $t_{1}$ in $\left[\alpha, t_{0}\right)$ such that $y_{i}^{\prime}\left(t_{1}\right)<0$. But from $Y^{\prime}=F Y$ we have $y_{i}^{\prime}\left(t_{1}\right) \geqq 0$. This contradiction completes the proof.

For the remainder of the paper we assume that $I=[a, b)$ where $a$ is finite or infinite and that $p$ has a continuous second derivative and $q$ a continuous first derivative on $I$.

In addition to the operator (1) and equation (2) we consider the formal adjoint operator $L^{+}$defined by

$$
L^{+} y=y^{\prime \prime \prime}-p y^{\prime \prime}+\left(q-2 p^{\prime}\right) y^{\prime}-\left(r-q^{\prime}+p^{\prime \prime}\right) y
$$

and the associated equation

$$
L^{+} y=0 .
$$

We are now ready to state our main results.

THEOREM 1. If $q \leqq 0$ and $r \leqq 0$, then equation (4) has property $\left(\mathrm{W}_{2}\right)$. 
Proof. Let $y$ be a solution of (2) whose initial values are all positive at $a$. Let $P=\int_{e}^{p}, Q=P q, R=P r$,

$$
Y=\left(\begin{array}{c}
y \\
y^{\prime} \\
P y^{\prime \prime}
\end{array}\right) \text { and } F=\left(\begin{array}{ccc}
0 & 1 & 0 \\
0 & 0 & 1 / P \\
-R & -Q & 0
\end{array}\right) \text {. }
$$

Then we have $Y^{\prime}=F Y$ and $Y(a)>0$. Hence, by Lemma $1, y(t)>0$ for $t>a$. From the result in [19] it follows that there exist first and second order linear differential operators $M$ and $N$ respectively, with leading coefficients nonzero on $I$, such that $L=N M$. Hence-see [13, p. 25]$L^{+}=M^{+} N^{+}$, where + denotes the formal adjoint operators. To get two solutions of $(4)$ having property $\left(\mathrm{W}_{2}\right)$ one can take any two linearly independent solutions of $\mathrm{N}^{+} y=0$ whose Wronskian is positive.

Since $\left(L^{+}\right)^{+}=L$, we can reverse the roles of equations (2) and (4) in the above argument to obtain the

COROLlaRY 1. If $q-2 p^{\prime} \leqq 0$ and $q^{\prime}-r-p^{\prime \prime} \leqq 0$, then equation (2) satisfies property $\left(\mathrm{W}_{2}\right)$.

THEOREM 2. If $q \leqq 0$ and $r \geqq 0$, then (2) has property $\left(\mathrm{W}_{2}\right)$.

Proof. Let $u$ and $v$ be the solutions of (2) determined by the initial conditions

$$
\begin{array}{lll}
y(a)=0, & y^{\prime}(a)=1, & y^{\prime \prime}(a)=0, \\
y(a)=0, & y^{\prime}(a)=0, & y^{\prime \prime}(a)=1
\end{array}
$$

respectively. Let $P, Q, R$ be as in the proof of Theorem 1. For

and

$$
y_{1}=\left(\begin{array}{ll}
u & v \\
u^{\prime} & v^{\prime}
\end{array}\right), \quad y_{2}=P y_{1}^{\prime}, \quad y_{3}=P\left(\begin{array}{ll}
u^{\prime} & v^{\prime} \\
u^{\prime \prime} & v^{\prime \prime}
\end{array}\right)
$$

$$
F=\left(\begin{array}{ccc}
0 & 1 / P & 0 \\
-Q & 0 & 1 \\
R & 0 & 0
\end{array}\right)
$$

we note that the vector $Y=\left[y_{1}, y_{2}, y_{3}\right]$ satisfies $Y^{\prime}=F Y$. The hypotheses imply $F(t) \leqq 0$.

Note that $y_{1}(a)=y_{2}(a)=0$ and $y_{3}(a)>0$. Hence

$$
y_{2}^{\prime}(a)=-Q(a) y_{1}(a)+y_{3}(a)>0 \text {. }
$$

So $y_{2}$ is increasing at $a$, hence $y_{2}$ is positive in a deleted right neighborhood $N$ of $a$. For $t$ in $N, y_{1}^{\prime}(t)>0$. Hence $y_{1}$ is also positive in $N$. Hence for $\alpha$ in $N$ we have $Y(\alpha)>0$. Therefore by Lemma $1, Y(t) \geqq 0$ for $t>a$. 
From $y_{1}^{\prime}(t)=[1 / P(t)] y_{2}(t) \geqq 0$ for $t>\alpha$ we have $y_{1}(t) \geqq y_{1}(\alpha)>0$ for $t>\alpha$. Since we already know $y_{1}(t)>0$ for $t$ in $N$ we may conclude that $y_{1}(t)>0$ for $t>a$. This concludes the proof.

By the result in [19], property $\left(\mathrm{W}_{2}\right)$ implies that the operator $L$ in (1) can be factored as: $L=M N$ where $M$ and $N$ are nonsingular differential operators of orders one and two respectively and where $u, v$ form a basis for the solution space of $N y=0$. The conclusions of Kim's Theorem 1 in [10] without the assumption of $p \leqq 0$ then follow from the fact that $u$ and $v$ are solutions of a second order equation.

A modification of the proof of Theorem 2 modeled after an argument of $\mathrm{Kim}[10]$ yields another result which we state as

COROLlaRY 2. If $q \leqq 0$ and $r \geqq 0$ then no nontrivial solution of (2) satisfies a boundary condition of the type

$$
y(c)=0 \text { and } y(d)=0=y^{\prime}(d) \text { for } a \leqq c<d<b .
$$

Proof. Suppose $u$ is a nontrivial solution of (2) satisfying (7). Multiplying by -1 , if necessary, we may assume $u^{\prime \prime}(d)>0$. Let $\alpha$ be the first number to the left of $d$ such that $u^{\prime \prime}(\alpha)=0$. Then $u(\alpha)>0$ and $u^{\prime}(\alpha)<0$. Let $v$ be the solution of (2) determined by the initial conditions $v(\alpha)=0$, $v^{\prime}(\alpha)=u(\alpha), v^{\prime \prime}(\alpha)=0$ and let $y=\left(\begin{array}{cc}u, & v \\ u^{\prime} & v^{\prime}\end{array}\right)$. Defining $Y$ as in the proof of Theorem 2 and arguing similarly we arrive at the conclusion $y(t)>0$ for $t>\alpha$. But this contradicts $y(d)=0$.

THEOREM 3. If $q \leqq 0, r \geqq 0, q-2 p^{\prime} \leqq 0$ and $q^{\prime}-p^{\prime \prime}-r \geqq 0$, then (2) is disconjugate.

Proof. The first two inequalities in the hypothesis imply, by Corollary 2 , that no nontrivial solution of equation (2) satisfies a boundary condition of type (7). Similarly the second pair of inequalities imply that no nontrivial solution of equation (4) satisfies a boundary condition of type (7). The conclusion then follows from the following known results in [1]: If (2) has a nontrivial solution with three zeros on $I$, then there is a nontrivial solution of (2) which satisfies a boundary condition of type (7) or of type

$$
y(c)=0=y^{\prime}(c) \text { and } y(d)=0 \text { with } a \leqq c<d<b .
$$

Furthermore, if (2) has a nontrivial solution satisfying (7) [(8)], then the adjoint equation (4) has a nontrivial solution satisfying (8) [(7)].

THEOREM 4. If $r-p q-q^{\prime} \geqq 0, q \leqq 0, q-2 p^{\prime} \leqq 0, p\left(q-2 p^{\prime}\right)+$ $\left(q^{\prime}-p^{\prime \prime}-r\right)+\left(2 p^{\prime}-q\right)^{\prime} \geqq 0$, then (2) is disconjugate.

Proof. Determine solutions $u, v$ of (2) by initial conditions (5) and (6). Define $P, Q, R$ as in the proof of Theorem 1 .

Let $y_{1}=W(u, v)$. We show that $y_{1}(t)>0$ for $t>a$. 
For $y_{2}=P y_{1}^{\prime}$, note that

$$
y_{2}^{\prime}=\left(P y_{1}^{\prime}\right)^{\prime}=P\left(\begin{array}{ll}
u^{\prime} & v^{\prime} \\
u^{\prime \prime} & v^{\prime \prime}
\end{array}\right)-Q y_{1}=y_{3} .
$$

Then the vector $Y=\left[y_{1}, y_{2}, y_{3}\right]$ satisfies $Y^{\prime}=F Y$ where

$$
F=\left(\begin{array}{ccc}
0 & 1 / P & 0 \\
0 & 0 & 1 \\
R-Q^{\prime} & -Q / P & 0
\end{array}\right)
$$

First we show that $y_{1}>0$ in some deleted right neighborhood of $a$. Note that $y_{1}(a)=y_{2}(a)=0$ and $y_{3}(a)=P(a)>0$. Now $y_{2}^{\prime}(a)=y_{3}(a)>0$ implies that $y_{2}$ is positive in some deleted right neighborhood $N$ of $a$. Hence $y_{2}>0$ and $y_{1}^{\prime}=(1 / P) y_{2}$ implies $y_{1}>0$ on $N$.

The first two inequalities in the hypothesis imply that $F(t) \geqq 0$. For $\alpha \in N$ we may conclude, from Lemma 1 , that $Y(t) \geqq 0$ for $t>\alpha$ and hence also $Y(t) \geqq 0$ for $t>a$. From $y_{j}(t) \geqq 0$ for $t>a, j=1,2,3$, and $y_{3}(a)>$ 0 , it follows that $y_{1}(t)>0$ for $t>a$.

Proceeding as in the proof of Corollary 2 we show that equation (2) has no nontrivial solution satisfying boundary condition (7). Similarly, using the third and fourth inequalities from the hypothesis, and arguing with respect to the adjoint equation (4) we show that (4) has no nontrivial solution satisfying boundary condition (7) for any $c$, $d$ in $I$ with $c<d$. The proof is then completed just as in Theorem 3 .

The factorization $L=M N$ is equivalent-see $\left[13\right.$, p. 25]-to $L^{+}=$ $N^{+} M^{+}$where + denotes the adjoint operator. In light of this, the conditions of Theorems 1 and 2 are sufficient for the factorization of $L^{+}$[the operator defined by the left-hand side of (3)] into a product of a second order operator times a first order one.

\section{REFERENCES}

1. N. V. Azbelev and Z. B. Caljuk, On the question of the distribution of the zeros of solutions of linear differential equations of third order, Mat. Sb. 51 (93) (1960), 475-486; English transl., Amer. Math. Soc. Transl. (2) 42 (1964), 233-245. MR 22 \#12266.

2. J. H. Barrett, Third-order differential equations with nonnegative coefficients, J. Math. Anal. Appl. 24 (1968), 212-224. MR 38 \#365.

3. - Oscillation theory of ordinary linear differential equations, Advances in Math. 3 (1969), 415-509. MR 41 \#2113.

4. J. M. Dolan, Oscillatory behavior of solutions of linear ordinary differential equations of third order, Doctoral Dissertation, University of Tennessee, Knoxville, Tenn., 1967 (unpublished).

5. A. M. Fink, An extension of Pólya's theorem, J. Math. Anal. Appl. 23 (1968), 625-627. MR 37 \#5470. 
6. M. Hanan, Oscillation criteria for third-order linear differential equations, Pacific J. Math. 11 (1961), 919-944. MR 26 \#2695.

7. P. Hartman, Principal solutions of disconjugate $n$-th order linear differential equations, Amer. J. Math. 91 (1969), 306-362. MR 40 \#450.

8. - On disconjugacy criteria, Proc. Amer. Math. Soc. 24 (1970), 374-381. MR $40 \# 4535$.

9. L. K. Jackson, Disconjugacy conditions for linear third-order differential equations, J. Differential Equations 4 (1968), 369-372. MR 37 \#1702.

10. W. J. Kim, Oscillatory properties of linear third order differential equations Proc. Amer. Math. Soc. 26 (1970), 286-293.

11. A. C. Lazer, The behavior of solutions of the differential equation $Y^{\prime \prime \prime}+p(x) Y^{\prime}+$ $q(x) Y=0$, Pacific J. Math. 17 (1966), 435-466. MR 33 \#1552.

12. A. Ju. Levin, The non-oscillation of solutions of the equation $x^{(n)}+p_{1}(t) x^{(n-1)}+$ $\cdots+p_{n}(t) x=0$, Uspehi Mat. Nauk 24 (1969), no. 2 (146), 43-96 = Russian Math. Surveys 24 (1969), no. 2, 43-99. MR 40 \#7537.

13. K. S. Miller, Linear differential equations in the real domain, Norton, New York, 1963.

14. Z. Nehari, Disconjugacy criteria for linear differential equations, J. Differential Equations 4 (1968), 604-611. MR 38 \#1329.

15. G. Pólya, On the mean-value theorem corresponding to a given Linear homogeneous differential equation, Trans. Amer. Math. Soc. 24 (1922), 312-324.

16. E. Willett, Asymptotic behavior of disconjugate Nth order differential equations, Canad. J. Math. 23 (1971).

17. D. Willett, Generalized de la Vallée Poussin disconjugacy tests for linear differential equations, Canad. Math. Bull. (to appear).

18. — Disconjugacy tests for singular linear differential equations (preprint).

19. Anton Zettl, Factorization of differential operators, Proc. Amer. Math. Soc. 27 (1971), 425-426.

Department of Mathematics, Northern Illinois University, DeKalb, Illinois 60115 